\title{
LW-MPCA-SVM-based Fault Diagnosis of Batch Process
}

\author{
Qing Yang ${ }^{1,2 *}$, Fenghai Miao ${ }^{3}$, Xu Zhang $^{1}$, Qingtao Wu ${ }^{1}$ \\ ${ }^{1}$ School of Information Science and Engineering, Shenyang Ligong University, Shenyang 110159, China \\ ${ }^{2}$ College of Optical and Electronical Engineering, Changchun University of Science and Technology, \\ Changchun 130022, China \\ ${ }^{3}$ Beijing Institute of Aerospace Control Devices, Beijing 100854, China \\ *Corresponding author's Email: yangqingxp@126.com
}

\begin{abstract}
An ensemble approach of fault diagnosis based on lifting wavelet, multi-way principal component analysis and support vector machines (LW-MPCA-SVM) for batch process is developed in this paper. Firstly, data are preprocessed to remove noise by lifting scheme wavelets, which are faster than first generation wavelets; then multi-way principal component analysis is used to extract feature for high-accurate diagnosis of the batch process, and SVM is used to diagnose faults. To validate the performance and effectiveness of the proposed scheme, LW-MPCA-SVM is applied to diagnose the faults in the simulation benchmark of fed-batch penicillin fermentation process. The results of simulation clearly demonstrate the effectiveness and feasibility of the proposed method, which diagnoses faults more accurately with desirable reliability.
\end{abstract}

Keywords: Fault diagnosis; Lifting wavelets; Multi-way principal component analysis; Support vector machines; LW-MPCA-SVM; Batch process

\section{Introduction}

Batch and transient operations are commonly used to manufacture high value-added products in the chemical, materials pharmaceutical, biological, and semiconductor industries. They are often characterized by the production of finite quantities of material by subjecting the input value-added products in the chemical, materials to a defined order of processing actions using one or more pieces of equipments. Process monitoring and fault diagnosis of such processes are extremely important to ensure high quality products and plant safety.

The common natures of non-steady, time- varying and non-linear behaviors make batch processes more difficult to diagnose than continuous processes. Process disturbances, which may vary with the development of time and from batch to batch, affect both pro- cess and product reproducibility. Paul Nomikos firstly developed the batch process monitoring approaches using multi-way principal component analysis (MPCA) [1]. Subsequently, several techniques using multivariate statistical analysis have been presented for the monitoring and fault diagnosis of batch processes [2-4]. But Hotelling's $T^{2}$ statistic and the statistic usually do not work well by only using normal MPCA when faults happen.

In early years, several statistical process control methods were well-recognized tools for on-line monitoring of the process status. Principal component analysis (PCA) proposed by Barry M. Wise [5] is primarily used in the area of chemistries. Nomikos and MacGregor [6, 7] presented a multi-way PCA method for batch process monitoring. $\mathrm{Ku}$, Storer, and Georgakis [8] proposed a dynamic PCA method by adding timelagged variables to augment the original data matrix in 
order to capture the dynamic characteristics. Furthermore, Shao, Jia, and Morris [9] integrated wavelets and non-linear PCA for non-linear process monitoring. Savita G. Kulkarni, Amit Kumar Chaudhary, Somnath Nandi, Sanjeev S. Tambe, Bhaskar D. Kulkarni [10] integrated PCA assisted generalized regression neural networks (GRNN). Lee, Yoo, and Lee [11] developed multiway kernel PCA for monitoring nonlinear batch processes. Nowadays, Xueqin Liu, Uwe Kruger, Tim Littler, Lei Xie, Shuqing Wang [12] presented moving window kernel PCA for adaptive monitoring of nonlinear processes.

As mentioned above, PCA has been successfully applied to multivariate process monitoring. However, PCA is limited to dealing with a linear continuous process, which can not be directly used in nonlinear batch process.

In this paper, an ensemble fault diagnosis method called LW-MPCA-SVM is put forward by using lifting wavelet transform as a preprocessor to reduce noise in data. SVM is a supervised learning method which requires no assumption of data structure and has been widely used for classification problems. There are many successful applications of SVM. For example, Shin, Eom, and Kim [13] applied one-class SVM for machine fault detection and classification. Shieh and Yang [14] applied fuzzy SVM to build classification model for product form design. Li [15] used SVM for copper clad laminate defects classification. For developing a successful SVM-based fault detector, the first step is feature extraction.

The rest part of this paper is organized as follows. The second section briefly reviews the concepts of lifting wavelets. The third section describes MPCA. And section 4 describes SVM. Then the batch diagnosis strategy based on LW-MPCA-SVM is presented in Section 5. The superiority of process monitoring and diagnosis using LW-MPCA-SVM in a simulation benchmark of fed-batch penicillin production is illustrated in Section 6. Finally, concluding remarks are made in Section 7.

\section{Lifting Scheme Wavelets}

The lifting scheme is a spatial domain construction of biorthogonal wavelets. The main feature of the lifting scheme is that it provides an entirely spatial domain interpretation of the transform, as opposed to the classical frequency domain-based constructions. In the decomposition procedure, the lifting scheme consists of three basic steps: split, prediction and update [16]. The steps of lifting scheme are shown in Figure

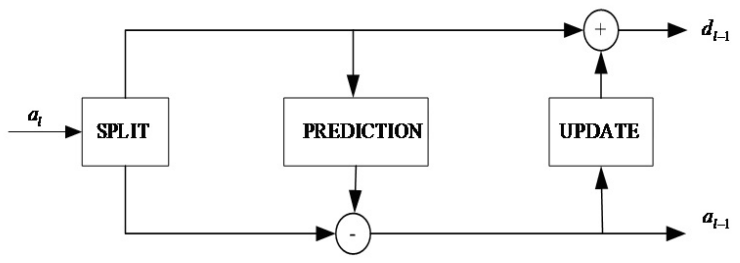

Figure 1 Lifting scheme wavelets process

1.

In the split step, the input samples $a_{l}$ are split into two disjoint samples: odd subset of samples $d_{l-1}$ and even sub set of samples $a_{l-1}$

$$
\left\{a_{l-1}, d_{l-1}\right\}=S\left(a_{l}\right)
$$

In the prediction step, as both sets of samples are highly correlative, the odd set of samples can be predicted by the even set of samples

$$
d_{l-1}=d_{l-1}-p\left(a_{l-1}\right),
$$

where $p=[p(0), \ldots, p(M-1)]$ is the prediction operator and $M$ is the length of $p$.

The update step is a primitive lifting process that offers better even set of samples. It aims at preserving the resolution characteristics of the input samples and obtaining a sub-sample.

$$
a_{l-1}=a_{l-1}-U\left(d_{l-1}\right),
$$

where $U=[U(0), \ldots, U(N-1)]$ is the update operator and $N$ is the length of $U$ [17].

Using signal $a_{l}$ as the input to lifting scheme can generate detail signal and approximation signal at lower resolution level.

\section{Multi-way Principal Component Analysis}

MPCA, based on PCA, is used to analyze batch process data. The experimental data can be constructed in the form of the three-dimensional array, as shown in Figure 2. A three-dimensional array of data matrix $X$ with $I \times J \times K$ summarizes a series of runs of a typical batch process, where $j=1,2 \ldots, J$ variables are measured at times $k=1,2 \ldots, K$ intervals throughout one batch. Similar data will be run at several numbers of batches $i=1,2 \ldots, I$. In MPCA, two way matrices (shown in Figure 2) should be constructed in order to perform the ordinary PCA. This means that the threedimensional array (measurements, batches and time) must be unfolded into a large two-dimensional matrix. The three dimensional array can be unfolded to put 


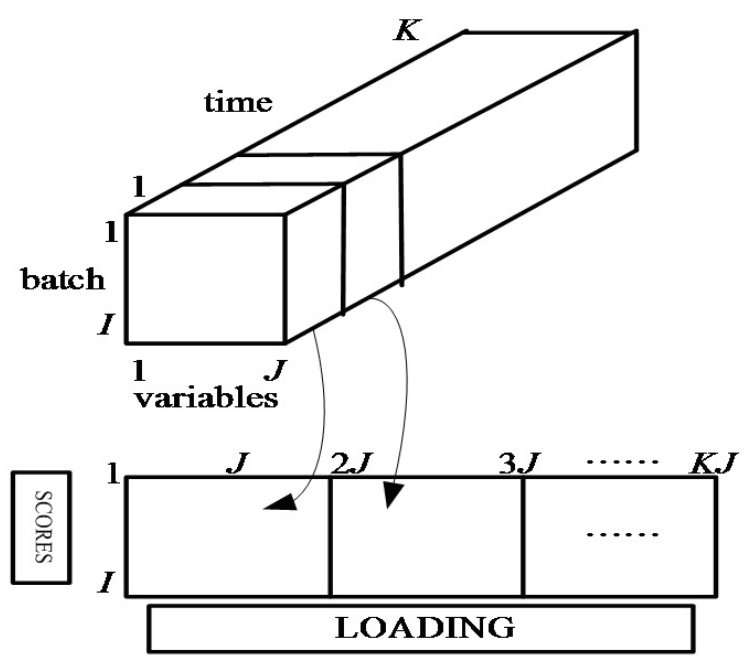

Figure 2 Two unfolding structures for MPCA

each vertical slice $I \times J$ side by side to the right, starting with the slice corresponding to the first time interval. The resulting two-dimensional matrix has dimensions $I \times K J$ [18]. PCA is used to decompose array $X$ into the summation of the product of score vectors $T$ and loading vectors $P$ :

$$
x=\sum_{r=1}^{R} t_{r} p_{r}+E=T P^{T}+E
$$

where $R$ is the number of principal components, $E$ is a residual matrix, and $T$ expresses the score matrix and is a loading matrix.

The distribution parameters at time are estimated from

$$
\begin{aligned}
& S P E_{k}=\left\{S P E_{1, k}, S P E_{2, k}, \ldots S P E_{I, k},\right\}, \\
& g^{k}=v^{k} / 2 m^{k}
\end{aligned}
$$

and

$$
h^{k}=2\left(m^{k}\right)^{2} / v^{k},
$$

where $m^{k}$ is the average of the $S P E_{k}$ and $v^{k}$ is the corresponding variance. Thus, SPE control limit at time $k$ can be approximated by

$$
S P E_{k, \alpha}=g^{k} \chi_{h^{k}, \alpha}^{2}=\frac{v^{k}}{2 m^{k}} \chi_{2\left(m^{k}\right)^{2} / v^{k}, \alpha}^{2}
$$

\section{Support Vector Machine Algorithm}

SVM is a supervised learning method used for classification and regression. The main idea behind this method is to map the data into higher dimensional input space where the different classes become linearly separable and to construct an optimal separating hyper-plane in this space [19].

Given a dataset points $\left\{\chi_{i}, d_{i}\right\}_{i=1}^{N}$ are given where $\chi_{i} \in R^{n}$ is the $i$ th input pattern and $d_{i} \in R$ is the $i$ th output pattern. For linearly separable data, SVM tries to determine a hyperplane that separates the data in the feature space. This plane can be represented by Eq. (6).

$$
f(\chi)=\omega^{T} \chi+b=0,
$$

where $\omega \in R^{n}$ is a weight vector and $b$ is a scalar. The vector $\chi$ and the scalar $b$ determine the position of the separating hyperplane. The classifier is constructed so that hyperplane satisfies the following inequalities for both the classes.

$$
\begin{aligned}
& \omega^{T} \phi\left(\chi_{i}\right)+b \geq+1, \quad \text { if } d_{i}=+1 \\
& \omega^{T} \phi\left(\chi_{i}\right)+b \leq-1, \quad \text { if } d_{i}=-1
\end{aligned}
$$

which is equivalent to

$$
d_{i}\left[\omega^{T} \phi\left(\chi_{i}\right)+b\right] \geq-1, \quad i=1,2, \ldots, N,
$$

where $\phi(\cdot)$ is a nonlinear function which maps the input space into a higher dimensional space. This function is not constructed explicitly. However, if a separating hyperplane does not exist in the higher dimensional space, slack variable $\xi_{i}$ is introduced so that

$$
\begin{aligned}
& d_{i}\left[\omega^{T} \phi\left(\chi_{i}\right)+b\right] \geq 1-\xi_{i}, \quad i=1,2, \ldots, N \\
& \xi_{i} \geq 0, \quad i=1,2, \ldots, N,
\end{aligned}
$$

To find optimal separating hyperplane, the risk bound is minimized according to the structural risk minimization principle by the optimization problem in Eq. (12).

$$
\min _{\omega, \xi_{i}} P_{1}\left(\omega, \xi_{i}\right)=\frac{1}{2} \omega^{T} \omega+c \sum_{i=1}^{N} \xi_{i},
$$

where $c$ is the regularization parameter which controls the tradeoff between complexity of the machine and the number of nonseparable point.

The aim of the SVM method is to construct a decision function for a classifier as following form.

$$
f(\chi)=\operatorname{sign}\left[\sum_{i=1}^{N} \alpha_{i} d_{i} K\left(\chi, \chi_{i}\right)+b\right],
$$

where $\alpha_{i}$ is positive real constants and $b$ is a real constant. Here $K(\cdot, \cdot)$ represents the kernel function. We have the following choices for kernel function [20]: 
1) Linear: $K\left(\chi, \chi_{i}\right)=\chi_{i}^{T} \chi$.

2) Polynomial (of degree $q$ ):

$$
K\left(\chi, \chi_{i}\right)=\left(1+\chi_{i}^{T} \chi\right)^{q}
$$

3) Radial basis function:

$$
K\left(\chi, \chi_{i}\right)=\exp \left(\frac{-\left|\chi-\chi_{i}\right|^{2}}{2 \sigma^{2}}\right),
$$

where $\sigma$ is a positive real number.

4) Two layer perceptron:

$$
K\left(\chi, \chi_{i}\right)=\tanh \left(\beta \chi_{i}^{T} \chi+\theta\right),
$$

where $\beta$ and $\theta$ are constants.

Therefore, we construct the Lagrangian function:

$$
\begin{aligned}
& J_{1}\left(\omega, v, \xi_{i} ; \alpha_{i}, v_{i}\right)=P_{1}\left(\omega, \xi_{i}\right)- \\
& \sum_{i=1}^{N} \alpha_{i}\left\{d_{i}\left[\omega^{T} \phi\left(\chi_{i}\right)+b\right]-1+\xi_{i}\right\} \\
& \quad-\sum_{i=1}^{N} v_{i} \xi_{i} b
\end{aligned}
$$

by introducing Lagrange multipliers

$$
\alpha_{i} \geq 0, v_{i} \geq 0(i=1,2, \ldots, N)
$$

By solving this, one obtains the following conditions

$$
\begin{aligned}
& \frac{\delta J_{1}}{\delta \omega}=0 \rightarrow \omega=\sum_{i=1}^{N} \alpha_{i} d_{i} \phi\left(\chi_{i}\right) \\
& \frac{\delta J_{1}}{\delta b}=0 \rightarrow \omega=\sum_{i=1}^{N} \alpha_{i} d_{i}=0 \\
& \frac{\delta J_{1}}{\delta \omega}=0 \rightarrow 0 \leq \alpha_{i} \leq c, i=1,2, \ldots N .
\end{aligned}
$$

This leads to the solution of the following quadratic programming problem:

$$
\begin{aligned}
& \max _{\alpha_{i}} Q_{1}\left(\alpha_{i} ; \phi\left(\chi_{i}\right)\right)=\sum_{i=1}^{N} \alpha_{i}- \\
& \frac{1}{2} \sum_{i=1}^{N} d_{i} d_{I} \phi\left(\chi_{i}\right)^{T} \phi\left(\chi_{I}\right) \alpha_{i} \alpha
\end{aligned}
$$

So that

$$
\sum_{i=1}^{N} \alpha_{i} d_{i}=0,0 \leq \alpha_{i} \leq c, i=1,2, \ldots N .
$$

The function $\phi\left(\chi_{i}\right)$ is related to $K\left(\chi, \chi_{i}\right)$ by imposing $\phi\left(\chi_{i}\right)^{T} \phi\left(\chi_{i}\right)=K\left(\chi, \chi_{i}\right)$, which is motivated by Mercer's theorem.

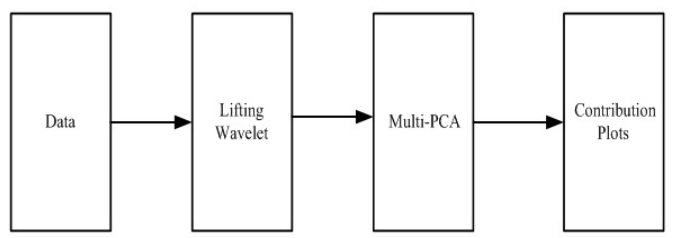

Figure 3 The sketch of monitoring based on LWPCA

Therefore, the classifier is designed by solving

$$
\begin{gathered}
\max _{\alpha_{i}} Q_{1}\left(\alpha_{i} ; K\left(\chi, \chi_{i}\right)\right)=\sum_{i=1}^{N} \alpha_{i}- \\
\frac{1}{2} \sum_{i=1}^{N} d_{i} d_{I} K\left(\chi, \chi_{i}\right) \chi_{i} \chi_{I}
\end{gathered}
$$

subject to

$$
\sum_{i=1}^{N} \alpha_{i} d_{i}=0,0 \leq \alpha_{i} \leq c, i=1,2, \ldots N .
$$

\section{LW-MPCA-SVM Algorithm}

\subsection{LW-MPCA algorithm}

The sketch of the proposed algorithm based on LWPCA is given in Figure 3.

The lifting wavelet transformation is performed for the process data and soft-threshold method is applied to the process detail signals at each level. As a result, the noise part in the wavelet coefficient is reduced.

Then MPCA is used to extract feature for high-accurate monitoring and/or diagnosis of the batch process. LWMPCA-based monitoring method is similar to PCAbased in that Hotelling's $T^{2}$ statistic in the feature space can be interpreted in the same way.

A measure of the variation within the PCA model is given by Hotelling's $T^{2}$ statistic, which is the sum of the normalized squared scores, and is defined as

$$
T^{2}=\left[t_{1}, \ldots, t_{p}\right] \wedge^{-1}\left[t_{1}, \ldots, t_{p}\right]^{T},
$$

where $\wedge^{-1}$ is the diagonal matrix of the inverse of the eigenvalues associated with the retained Principal Components (PCs). The confidence limit for $T^{2}$ is obtained by using the $F$-distribution:

$$
T_{p, N, \alpha}^{2} \sim \frac{p(N-1)}{N-p} F_{p, N-p, \alpha},
$$

where $N$ is the number of samples in the model and $p$ is the number of PCs [18]. 


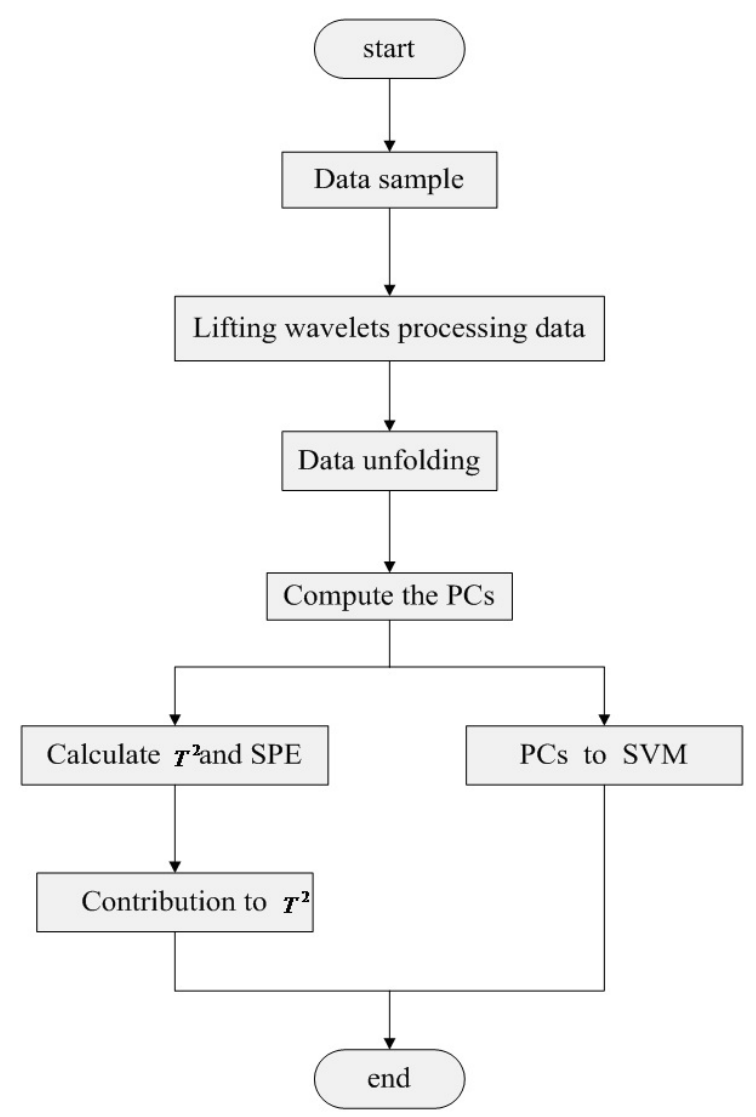

Figure 4 LW-MPCA-SVM algorithm

The $S P E$ is defined as the sum of the squares of $e_{j k}$ :

$$
Q=\sum_{j k=1}^{J K} Q_{j k}=\sum_{j k=1}^{J K} e_{j k}^{2},
$$

Thus $e_{j k}^{2}$ is the contribution of variable $j$ at sample $k$ to the SPE statistic [21].

Contribution plot is available for fault diagnosis. Contribution plots for the SPE statistic show which variable is the root cause of the fault. The process variable with the highest contribution is possibly the cause of the abnormality.

\subsection{LW-MPCA- SVM algorithm}

The steps of LW-MPCA-SVM algorithm are summarized in Figure 4

\section{Case Study}

The proposed scheme is applied to a modular simulator for fed-batch penicillin fermentation process, which was developed by G. Birol, C. Ündey and A. Çinar [22]. The modular is used for comparing the various monitoring and control methods used in batch process. This process utilizes a special microorganism that is being propagated under special condition to

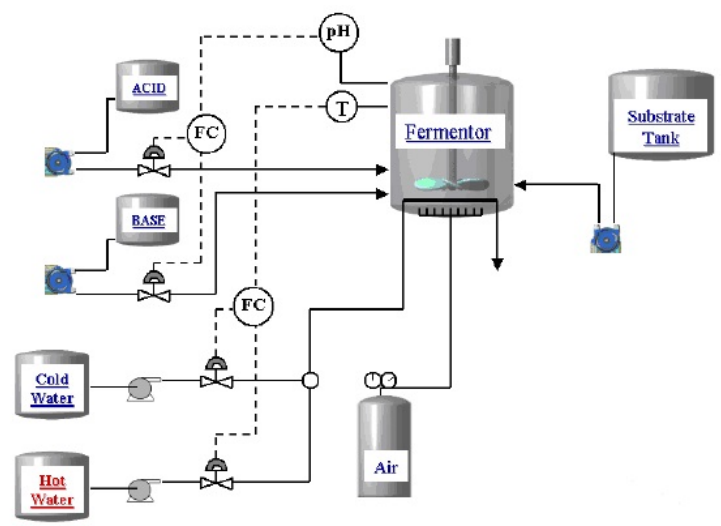

Figure 5 Flowsheet of Pensim simulator

produce antibiotic as the product of secondary metabolites. This benchmark model is capable of simulating concentrations of biomass, $\mathrm{CO}_{2}$, penicillin, carbon source, oxygen and heat generation under various operating conditions. These simulations are run under closed-loop control of $\mathrm{pH}$ and temperature, whereas, glucose addition is performed open looped [23]. A flow diagram of the penicillin fermentation processes is given in Figure 5. More information on this modular can be obtained from the website of the monitoring and control Group of the Illinois Institute of technology.

Training data sets (50 batches) and one set are produced by running the simulator repeatedly under normal operating conditions with small random variations in the initial setting values. Ten variables are selected for monitoring the process; those variables are listed in Table 1. The typical time-profiles of ten variables are shown in Figure 6.

To simulate the process noise under real industrial condition, the values of process variables are corrupted by using independently Gaussian white noise with zero mean and standard deviation of 0.1 . The duration of each batch is $400 \mathrm{~h}$ and the sampling interval is chosen

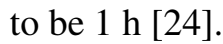

In the batch process, a fault, correspondent to variable 3 , is introduced into the process. The fault is $20 \%$ step increased. The substrate feed rate influences the production of the penicillin. It consequently decreases the penicillin productivity [25]. The process monitoring results by MPCA and LW-MPCA are shown in Figure 7 and Figure 8.

For step fault as the first case, Hotelling's $T^{2}$ statistic and the SPE statistic detect the fault at the same time. With respect to ramp fault, LW-MPCA detects the faults significantly more efficient and slightly bet- 


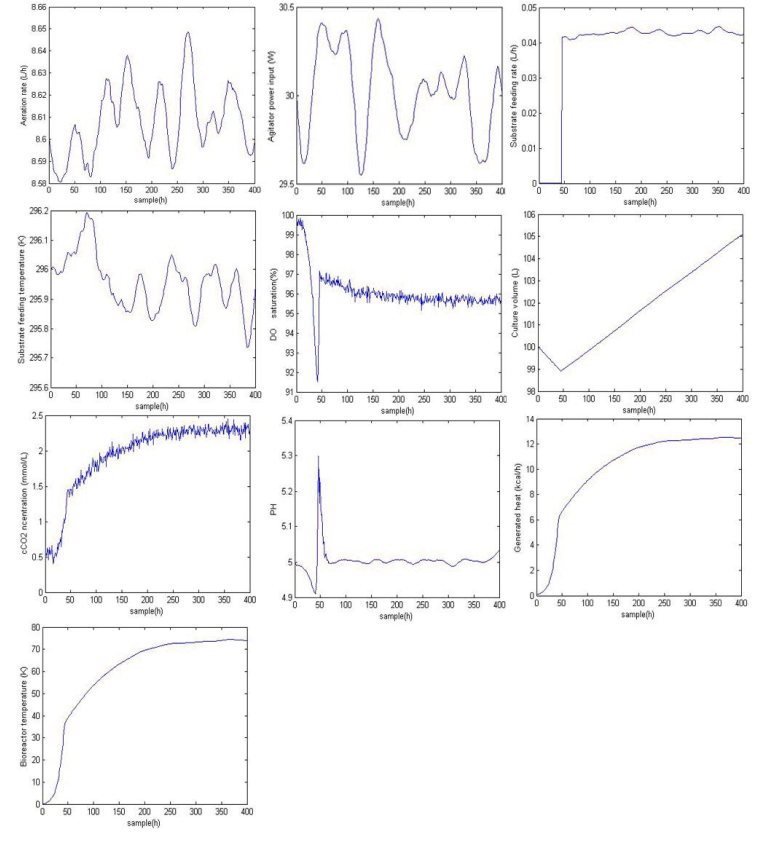

Figure 6 Process variables

ter than MPCA. In addition, the results of different numbers of the reference batches in the example of benchmark fed-batch penicillin fermentation demonstrate that the false alarm rate in LWMPCA monitoring is more accurate than that of MPCA in the case of smaller reference datasets.

In this experiment, the LW-MPCA-SVM approach is applied to the batch process. LW-MPCA-SVM is compared with SVM, and the experiment results are shown in Figure 9-14. In the simulation experiment three faults are set up and shown in Table 2. The accurate diagnosis rates of the two methods are shown in Table 3, and the dimension reduced by PCA in Figure $15-17$

Table 1 Variables used in the monitoring of the benchmark fed-batch penicillin fementation process

\begin{tabular}{|c|c|}
\hline Number & Process variables \\
\hline 1 & Aeration rate $(\mathrm{L} / \mathrm{h})$ \\
\hline 2 & Agitator power input $(\mathrm{W})$ \\
\hline 3 & Substrate feeding rate $(\mathrm{L} / \mathrm{h})$ \\
\hline 4 & Substrate feeding temperature $(\mathrm{K})$ \\
\hline 5 & saturation $(\%)$ \\
\hline 6 & Culture volume $(\mathrm{L})$ \\
\hline 7 & $\mathrm{CO}_{2}$ concentration $(\mathrm{mmol} / \mathrm{L})$ \\
\hline 8 & $\mathrm{pH}$ \\
\hline 9 & Bioreactor temperature $(\mathrm{K})$ \\
\hline 10 & Generated heat $(\mathrm{kcal} / \mathrm{h})$ \\
\hline
\end{tabular}
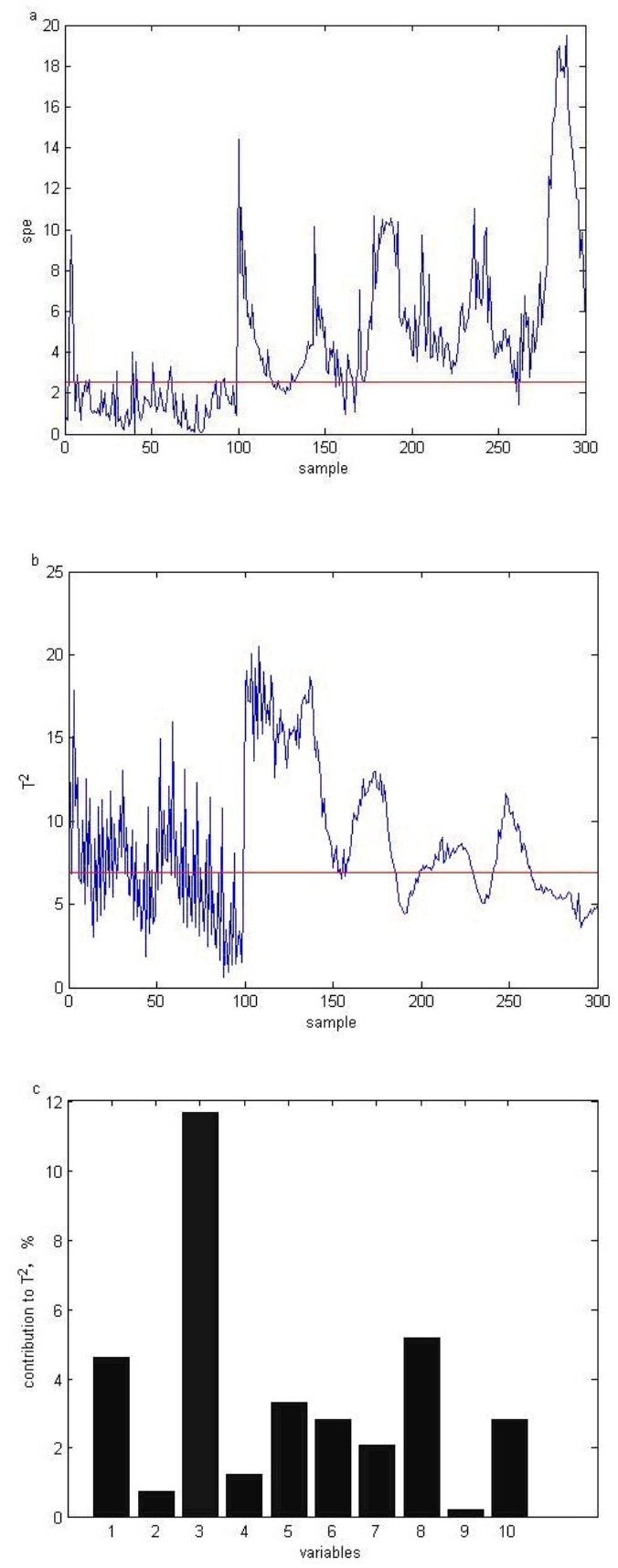

Figure 7 MPCA process monitoring

Table 2 Variables used in the monitoring of the benchmark fed-batch penicillin fementation process

\begin{tabular}{|c|c|}
\hline Number & Fault Description \\
\hline Fault 1 & $\begin{array}{c}\text { Substrate feed flow rate is suddenly } \\
\text { step-increased by } 10 \% \text { at } 250 \mathrm{~h} \text { and } \\
\text { maintained to } 400 \mathrm{~h} \text { of batch operation }\end{array}$ \\
\hline Fault 2 & $\begin{array}{c}\text { Aeration rate is linearly increased by } 30 \% \\
\text { with the ramp rate from } 200 \mathrm{~h} \text { to the end of } \\
\text { batch operation }(400 \mathrm{~h})\end{array}$ \\
\hline Fault 3 & $\begin{array}{c}\text { Substrate feed flow rate is step-increased by } \\
90 \% \text { at } 10 \mathrm{~h} \text { and maintained to } 400 \mathrm{~h} \text { of batch } \\
\text { operation }\end{array}$ \\
\hline
\end{tabular}



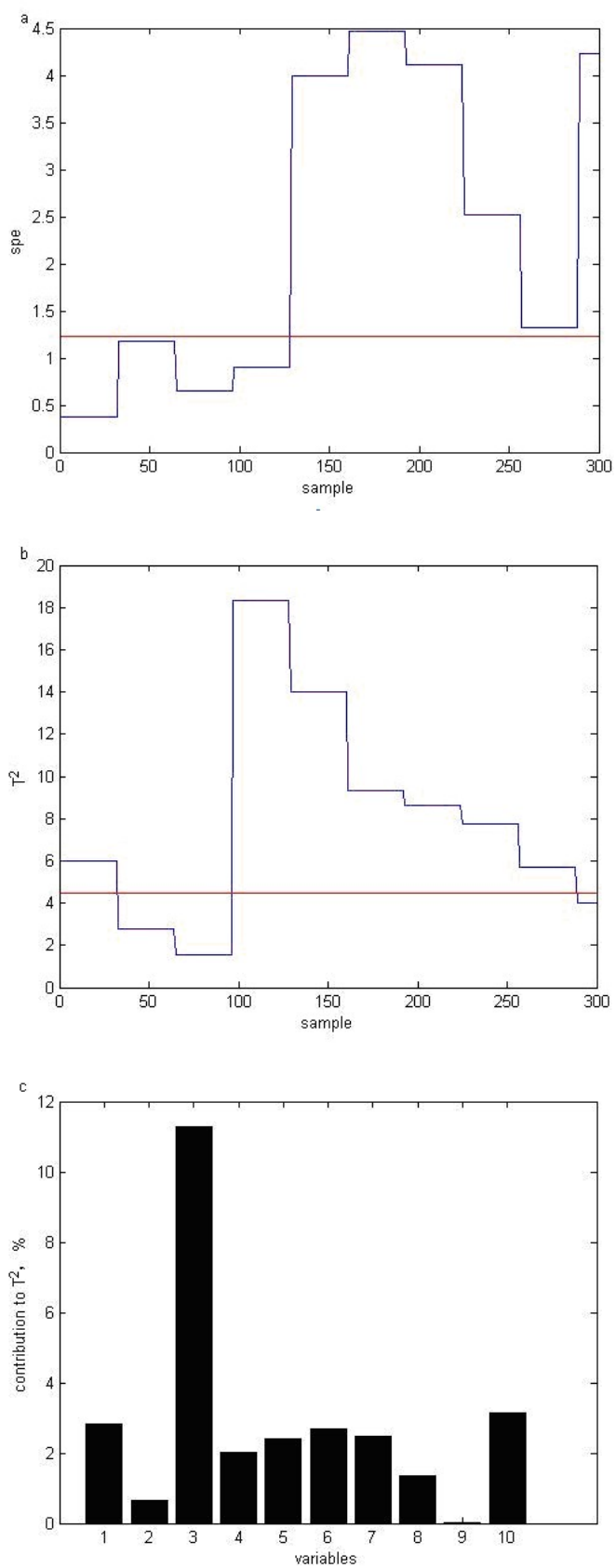

Figure 8 LWMPCA process monitoring

Table 3 Comparative analysis of the faults diagnosis rates by the three diagnosis methods (\%)

\begin{tabular}{|c|ccc|}
\hline Fault Type & Fault 1 & Fault 2 & Fault 3 \\
\hline LW-MPCA-SVM & 99.13 & 97.25 & 99.75 \\
\hline SVM & 62.50 & 94.63 & 99.88 \\
\hline
\end{tabular}

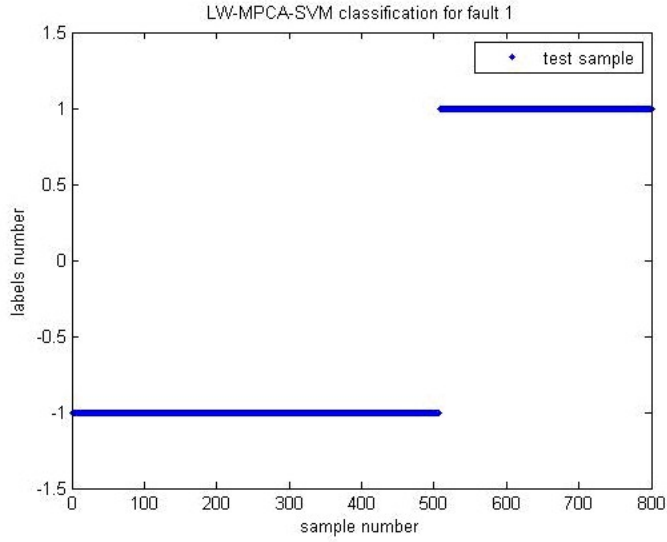

Figure 9 LW-MPCA-SVM classification for fault 1

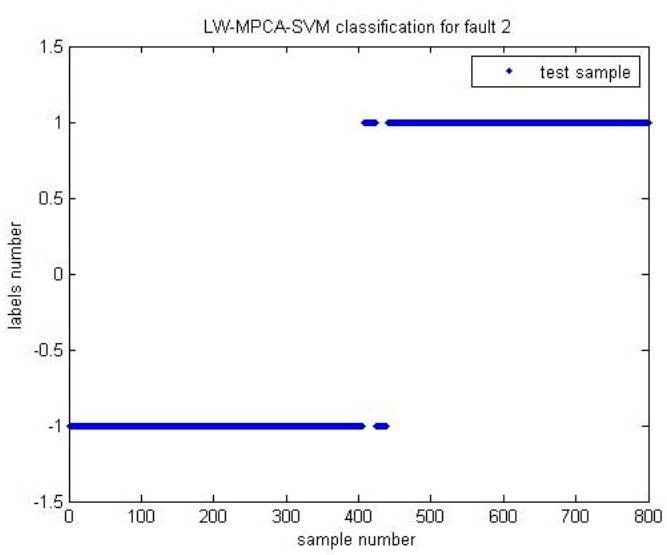

Figure 10 LW-MPCA-SVM classification for fault 2

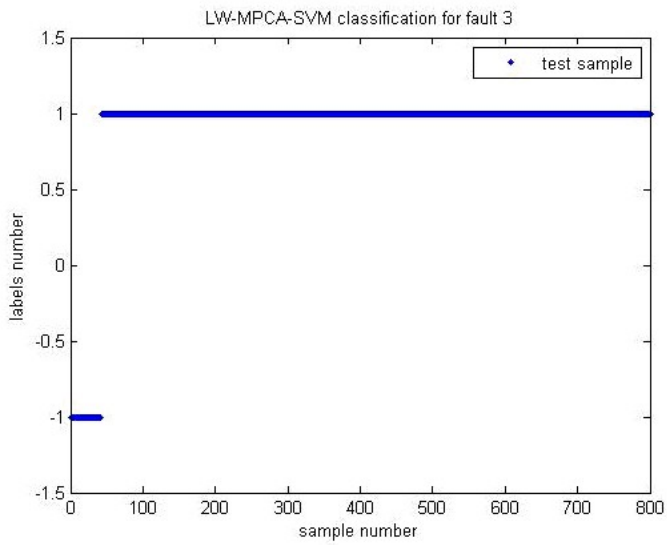

Figure 11 LW-MPCA-SVM classification for fault 3 


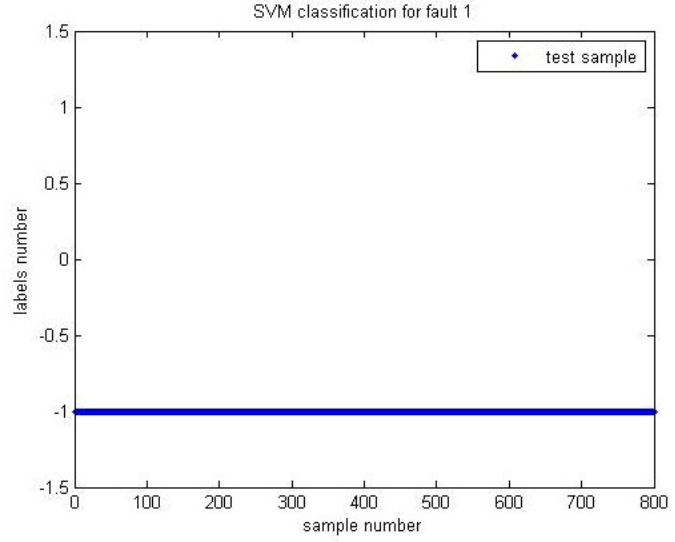

Figure 12 SVM classification for fault 1

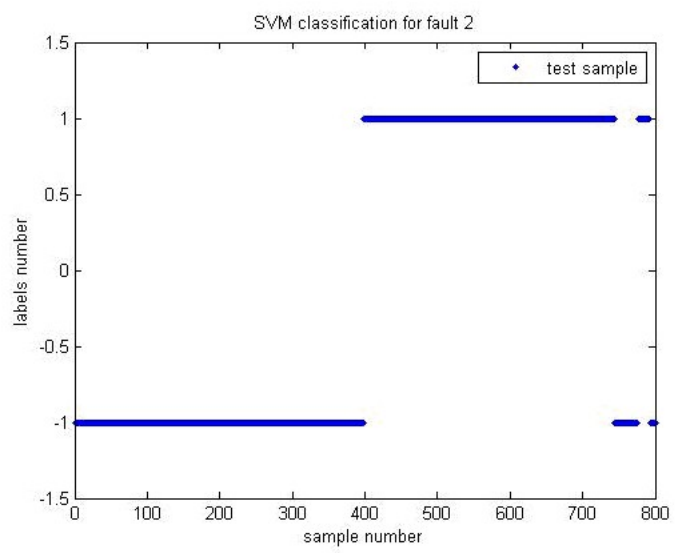

Figure 13 SVM classification for fault 2

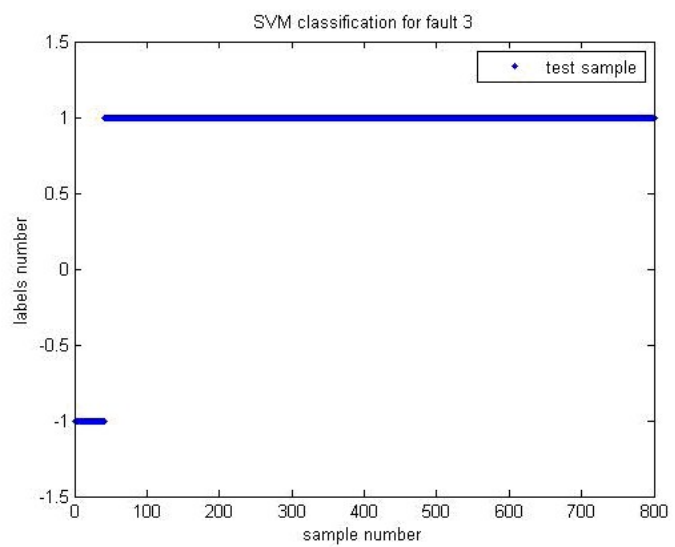

Figure 14 SVM classification for fault 3

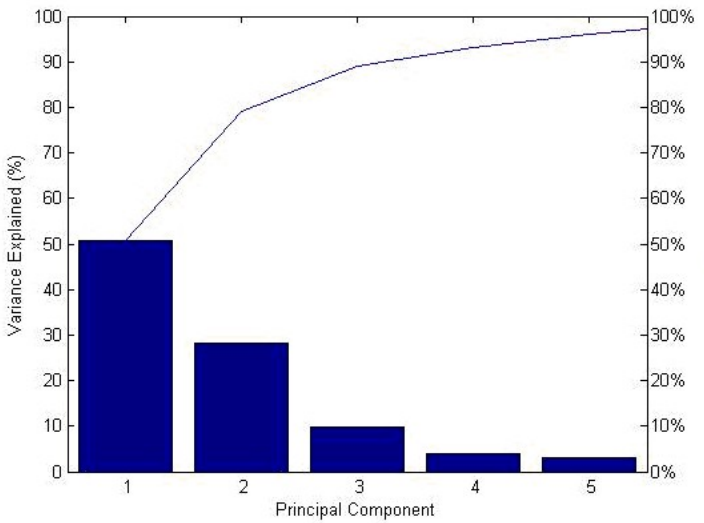

Figure 15 Dimensionality reduction for fault 1

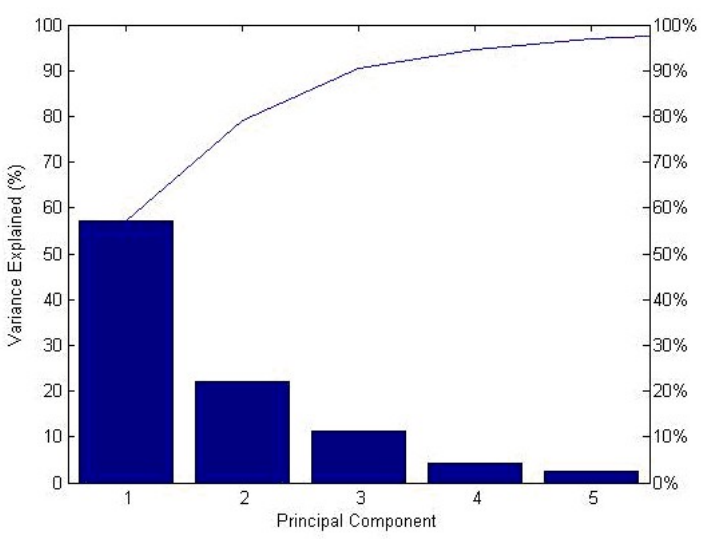

Figure 16 Dimensionality reduction for fault 2

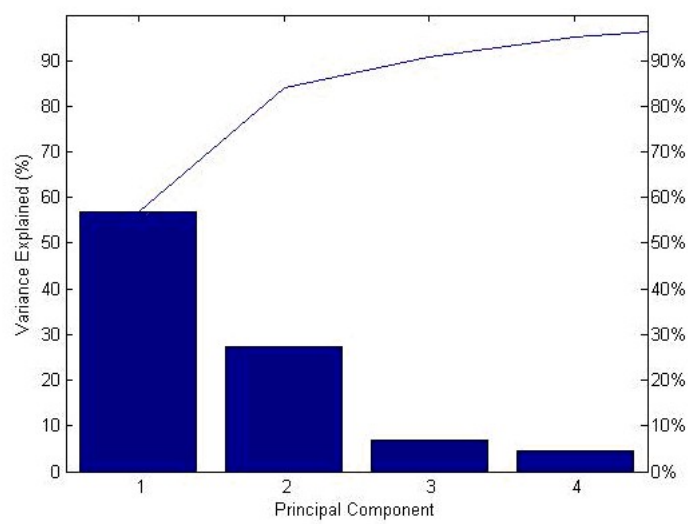

Figure 17 Dimensionality reduction for fault 3 


\section{Conclusion}

In this paper, we have proposed an ensemble LWMPCA-SVM approach for fault diagnosis of batch process. The noise in data is reduced by the lifting wavelet scheme. Then multi-way principal component analysis is used to extract feature for high-accurate monitoring and/or diagnosis of the batch process. Finally, SVM is used to diagnose faults. Simulations conducted on the penicillin fermen-tation model have shown that LW-MPCA is more efficient and has better performance than the MPCA. And LW-MPCA-SVM algorithm for classification of batch process is more efficient than SVM and gives better recognition accuracy.

\section{References}

[1] Paul Nomikos, "Detection and diagnosis of abnormal batch operations based on multi-way principal component analysis", In: World Batch Forum, Toronto,Canada, ISA Transactions, Vol.5, pp.259266. 1996.

[2] Xuan-Tien Doan and Rajagopalan Srinivasan, "Online monitoring of multi-phase batch processes using phase-based multivariate statistical process control", Computers \& Chemical Engineering, Vol.32, pp.230-243, 2008.

[3] Yuan Yao and Furong Gao, "Phase and transition based batch process modeling and online monitoring”, Journal of Process Control, Vol.19, pp. 816826, 2009.

[4] Ji-Dong Shao, Gang Rong, and Jong Min Lee, "Learning a data-dependent kernel function for KPCA-based nonlinear process monitoring", Chemical Engineering Research and Design, Vol.87, pp.1471-1480, 2009.

[5] Barry M. Wise and Neal B. Gallagher, "The process chemometrics approach to process monitoring and fault detection", Journal of Process Control, Vol.6, pp.329-348, 1996.

[6] P. Nomikos and J. F. MacGregor, "Monitoring batch processes using multiway principal component analysis", American Institute of Chemical Engineers Journal, Vol.40, pp.1361-1375, 1994.

[7] P. Nomikos and J. F. MacGregor, "Multivariate SPC charts for monitoring batch processes", Technometrics, Vol.37, pp.41-59, 1995.

[8] Wenfu Ku, Robert H. Storer,and Christos Georgakis, "Disturbance detection and isolation by dynamic principal component analysis", Chemometrics and Intelligent Laboratory Systems, Vol.30, pp.179-196, 1995.
[9] R. Shao, F. Jia, E. B. Martin, and A. J. Morris, "Wavelets and non-linear principal components analysis for process monitoring", Control Engineering Practice, Vol.7, pp.865-879, 1999.

[10] Savita G. Kulkarni, Amit Kumar Chaudhary, Somnath Nandi, Sanjeev S. Tambe, and Bhaskar D. Kulkarni, "Modeling and monitoring of batch processes using principal component analysis (PCA) assisted generalized regression neural networks(GRNN)", Biochemical Engineering Journal, Vol.18, pp.193-210, 2004.

[11] J. M. Lee, C. K. Yoo, and I. B. Lee, "Fault detection of batch processes using multiway kernel principal component analysis", Computers and Chemical Engineering, Vol.28, pp.1837-1847, 2004.

[12] Xueqin Liu, Uwe Kruger, Tim Littler, Lei Xie, and Shuqing Wang, "Moving window kernel PCA for adaptive monitoring of nonlinear processes", Chemometrics and Intelligent Laboratory Systems, Vol.96, pp.132-143, 2009.

[13] Shin, H. J., Eom, D. H., \& Kim, S. S., "One-class support vector machines-an application in machine fault detection and classification", Computers \& Industrial Engineering, Vol.48, pp.359-408, 2005.

[14] X. Song and A. M. Wyrwicz, "Unsupervised spatiotemporal fMRI data analysis using support vector machines", NeuroImage, Vol.47, pp.204-212, 2009.

[15] T. S. Li, "Applying wavelets transform and support vector machine for copper clad laminate defects classification", Computers \& Industrial Engineering, Vol.56, pp.1154-1168, 2009.

[16] Yuna Pan, Jin Chen, and Xinglin Li, "Bearing performance degrada-tion assessment based on lifting wavelet packet decomposition and fuzzy c-means", Mechanical Systems and Signal Processing, Vol.24, pp.559-566, 2010.

[17] Wen Bao, Rui Zhou, Jianguo Yang, DarenYu, and Ning Li, "Anti-aliasing lifting scheme for mechanical vibration fault feature extraction", Mechanical Systems and Signal Processing, Vol.23, pp.1458-1473, 2009.

[18] Kunlun Hu and Jingqi Yuan, "Batch process monitoring with tensor factorization", Journal of Process Control, Vol.19, pp.288-296, 2009.

[19] Samit Ari, Koushik Hembram and Goutam Saha, "Detection of cardiac abnormality from PCG signal using LMS based east square SVM classifier", Expert Systems with Applications, Vol.37, pp.80198026, 2010.

[20] J. A. K. Suykens and J. Vandewalle, "Least square support machine classifiers", Neural Processing Letters, Vol.9, pp.293-300, 1999. 
[21] Mingxing Jia, Fei Chu, Fuli Wang, and Wei Wang, "On-line batch process monitoring using batch dynamic kernel principal component analysis", Chemometrics and Intelligent Laboratory Systems, Vol.101, pp.110-122, 2010.

[22] Gülnur Birol, Cenk Ündey and Ali Çinar, “A modular simulation package for fed-batch fermentation penicillin production", Computers \& Chemical Engineering, Vol.26, pp.1553-1565, 2002.

[23] Xi Zhang, Weiwu Yan, Xu Zhao, and Huihe Shao., "Nonlinear bio-logical batch process monitoring and fault identification based on kernel fisher discriminant analysis", Process Biochemistry, Vol.42, pp.1200-1210, 2007.

[24] Xuemin Tian, Xiaoling Zhang, Xiaogang Deng, and Sheng Chen., "Multi-way kernel independent component analysis based on feature samples for batch process monitoring", Neurocomputing, Vol.72, pp.1584-1596, 2009.

[25] Wang Zhifeng and Yuan Jingqi., "Online supervision of penicillin cultivations based on rolling MPCA", Chinese Journal of Chemical Engineering, Vol.15, pp.92-96, 2007. 